\title{
Albuminúria e fatores associados a doença renal crônica na obesidade
}

\author{
Albuminúria and factors associated with chronic kidney disease in obesity \\ Albuminúria y factores asociados a enfermedad renal crónica en obesidad
}

\section{Resumo}

Introdução: A albuminúria é um importante marcador para o aumento da morbimortalidade em obesos e um parâmetro de rastreio para alterações renais precoces. No entanto, poucos estudos associam o impacto da obesidade na albuminúria. Objetivo: Comparar a albuminúria e fatores de risco para doença renal crônica com a obesidade. Métodos: Estudo transversal com obesos, maiores de 18 anos, sem diagnóstico de doença renal crônica, alocados de acordo com o Índice de Massa Corporal, nos grupos A (30-39,99 kg/m² e 40-49 kg/m²) e B ( $\geq 50 \mathrm{~kg} / \mathrm{m}^{2}$ e $\left.\geq 60 \mathrm{~kg} / \mathrm{m}^{2}\right)$. A avaliação renal ocorreu através da aferição de albuminúria, ureia e creatinina. Também foram avaliados perfil lipídico, inflamatório e glicêmico. Foram realizados os testes qui-quadrado, t student, Mann-Whitney e correlação de Spearman, com auxílio do software Statistical Package for the Social Sciences. Os resultados foram analisados considerando nível de significância com p<0,05 e intervalo de confiança de 95\%. Resultados: 97 obesos obtiveram frequência de albuminúria de $21,6 \%$, sem diferença estatística entre os grupos. Dos fatores de risco, observou-se que os obesos >40 anos apresentaram maior prevalência de hipertensão. Os níveis séricos diminuídos de HDL e aumentados de PCR quando relacionados ao Índice de Massa Corporal apresentaram $\mathrm{p}<0,05$. A albuminúria apresentou fraca correlação com idade e índice de massa corporal $(0,031$ e 0,136 , respectivamente). Conclusão: A frequência de albuminúria foi elevada, mas não se associou com a gravidade da obesidade. Os obesos adultos de meia idade apresentaram mais fatores de risco para danos renais.

Palavras-chave: Proteinúria; Albuminúria; Insuficiência renal crônica; Fatores de risco; Cirurgia bariátrica; Obesidade mórbida.

\footnotetext{
Abstract

Introduction: Albuminuria is an important marker for increased morbidity and mortality in obese and a screening parameter for early renal changes. However, few studies associate the impact of obesity on albuminuria. Objective: To relate albuminuria and risk factors for chronic kidney disease with obesity. Methods: Cross-sectional study with obese patients, over 18 years old, without a diagnosis of chronic kidney disease, allocated according to the Body Mass Index, in groups A $\left(30-39,99 \mathrm{~kg} / \mathrm{m}^{2}\right.$ and $\left.40-49 \mathrm{~kg} / \mathrm{m}^{2}\right)$ and $B\left(\geq 50 \mathrm{~kg} / \mathrm{m}^{2}\right.$ and $\left.\geq 60 \mathrm{~kg} / \mathrm{m}^{2}\right)$. Renal evaluation occurs through the measurement of albuminuria, urea and creatinine. Lipid, inflammatory and glycemic profile were also evaluated. Chi-square, student t, Mann-Whitney and Spearman correlation tests were performed, with the Statistical Package for the Social Sciences software. The results were achieved with a significance level of $\mathrm{p}<0,05$ and a $95 \%$ confidence interval. Results: 97 obese individuals had an albuminuria frequency of $21.6 \%$, with no statistical difference between the groups. The decreased serum levels of HDL and increased CRP when related to the additional body mass index $\mathrm{p}<0,05$. Of the risk factors, it was observed that the obese $>40$ years old had a higher prevalence of hypertension. Albuminuria has a low correlation with age and body mass index $(0,031$ and 0,136 , respectively).
} 
Conclusion: The frequency of albuminuria was high, but it was not associated with the severity of obesity. Obese middle-aged adults have more risk factors for kidney damage.

Keywords: Proteinuria; Albuminuria; Chronic renal insufficiency; Risk factors; Bariatric surgery; Morbid obesity.

\section{Resumen}

Introducción: La albuminuria es un marcador importante de aumento de la morbilidad y mortalidad en obesos y un parámetro de cribado de cambios renales precoces. Sin embargo, pocos estudios asocian el impacto de la obesidad en la albuminuria. Objetivo: comparar la albuminuria y los factores de riesgo de enfermedad renal crónica con la obesidad. Métodos: Estudio transversal con obesos, mayores de 18 años, sin diagnóstico de enfermedad renal, asignados según el Índice de Masa Corporal, en los grupos A $\left(30-39,99 \mathrm{~kg} / \mathrm{m}^{2}\right.$ y $\left.40-49 \mathrm{~kg} / \mathrm{m}^{2}\right)$ y B $\left(\geq 50 \mathrm{~kg} / \mathrm{m}^{2}\right.$ y $\geq 60$ $\mathrm{kg} / \mathrm{m}^{2}$ ). La evaluación renal se realizó midiendo albuminuria, urea y creatinina. También se evaluaron los perfiles lipídicos, inflamatorios y glucémicos. Se realizaron las pruebas de correlación de chi-cuadrado, t de Student, MannWhitney y Spearman, utilizando el software Statistical Package for the Social Sciences. Los resultados se analizaron considerando un nivel de significancia con p <0,05 y un intervalo de confianza del 95\%. Resultados: 97 obesos tuvieron una frecuencia de albuminuria del 21,6\%, sin diferencia estadística entre grupos. De los factores de riesgo, se observó que las personas obesas > 40 años tenían una mayor prevalencia de hipertensión. Los niveles séricos disminuidos de HDL y los niveles aumentados de PCR cuando se relacionan con el Índice de Masa Corporal presentaron $\mathrm{p}<0,05$. La albuminuria se correlacionó débilmente con la edad y el índice de masa corporal $(0,031$ y 0,136, respectivamente). Conclusión: la frecuencia de albuminuria fue alta, pero no se asoció con la gravedad de la obesidad. Los adultos obesos de mediana edad tenían más factores de riesgo de daño renal.

Palabras clave: Proteinuria; Albuminuria; Insuficiencia renal crónica; Factores de riesgo; Cirugía bariátrica; Obesidad morbida.

\section{Introdução}

A obesidade é definida como excesso de adiposidade em consequência a um estado prolongado de balanço energético positivo e caracterizada por um estado de inflamação crônica de baixo grau (Engin \& Engin, 2017).

A sobrecarga lipídica leva a disfuncionalidade do tecido adiposo (TA), desencadeando processos de remodelação celular e estrutural, hipertrofia adipocitária, aumento da fibrose e comprometimento da função e estrutura vascular (Pellegrinelli, Carobbio \& Vidal-Puig, 2016). Essas alterações afetam a homeostase metabólica, levando ao acúmulo de gordura ectópica, contribuindo para o aparecimento de comorbidades relacionadas à obesidade (Unamuno et al. 2018).

O método mais comum para definir a obesidade é baseado no Índice de Massa Corporal (IMC), onde o peso de uma pessoa, em quilogramas, é dividido pelo quadrado de sua altura, em metros. De acordo com a Organização Mundial da Saúde o peso normal é considerado a partir do IMC 20 e $25 \mathrm{~kg} / \mathrm{m}^{2}$, o excesso de peso (Stenvinkel, Zoccali \& Ikizler, 2013; WHO, 2004) entre o IMC 25 e $30 \mathrm{~kg} / \mathrm{m}^{2}$ e obesidade a partir do IMC $\geq 30 \mathrm{~kg} / \mathrm{m}^{2}$.

O IMC elevado é um dos mais fortes fatores de risco para o desenvolvimento de doença renal crônica (DRC) (Kovesdy, Furth \& Zoccali, 2017), provavelmente por associação aos componentes da síndrome metabólica, compressão renal e dos mecanismos biológicos derivados do excesso de TA (Who, 2004). Além disso, o tempo de obesidade por si, mesmo sem levar em consideração a presença de comorbidades como HAS e DM, é um importante fator de risco para danos renais, como o desenvolvimento da albuminúria (Pinto-Sietsma et al.2003; Ejerblad et al. 2006). A DRC é definida como anormalidades da estrutura ou função renal, presentes por um período maior que 3 meses com implicações para saúde (Chagnac et al. 2000).

Obesos graves apresentam piores desfechos renais, já que evoluem precocemente com hiperfiltração e glomerulomegalia (Herrington et al. 2017; Yazdani et al. 2019) mecanismo este adaptativo que leva a glomeruloesclerose segmentar e focal secundária. Culminando com proteinúria e piora da função renal, o que aumenta risco de evolução para DRC estágio 5 e necessidade de terapia renal substitutiva (Chagnac et al. 2000; Serra et al. 2008).

Um importante marcador para o aumento da morbimortalidade em obesos é a albuminúria (Valensi et al. 1996; Bastos, Bregman \& Kirsztajn, 2010; Minoo, Mahdavi-Mazdeh, Abbasi \& Sohrabi, 2015), parâmetro que reflete a excreção urinária de albumina quando a quantidade está acima normal, mas aquém dos níveis detectados nos testes de proteinúria total (Bastos, Bregman \& Kirsztajn, 2010). Porém, sua mensuração é pouco solicitada na prática clínica como tal, para os diversos 
grupos de indivíduos em risco de desenvolvimento de DRC (Zanella, 2006).

Diante do exposto, considerando que são limitados dados sobre a progressão da DRC nos estágios iniciais, principalmente comparando a gravidade de obesidade e grau de albuminúria (Stenvinkel, Zoccali \& Ikizler, 2013), é imprescindível identificar pacientes em risco para prevenir ou tratar precocemente a nefropatia ainda no estágio inicial.

O presente estudo objetivou relacionar a albuminúria aumentada e fatores de risco para DRC na obesidade.

\section{Metodologia}

\section{Características do estudo}

Utilizou-se um delineamento transversal observacional com obesos do Programa de Cirurgia Bariátrica e Ambulatório de Nutrição Clínica do Hospital Universitário Professor Alberto Antunes (HUPAA), em Maceió, Alagoas. O recrutamento ocorreu aleatoriamente por conveniência, no período de maio de 2018 a novembro de 2019. Foram incluídos pacientes de ambos os sexos, maiores de 18 anos, sem diagnóstico de DRC. Não foram incluídos portadores de HIV, pacientes com positividade aos vírus da hepatite B e C, portadores de câncer, e indivíduos com lesão renal aguda (LRA), gestantes, alcoólatras.

\section{População}

Foram entrevistados 161 pacientes, destes, 7 não foram incluídos no estudo, 3 por serem cadeirantes e não conseguirem realizar a antropometria, 1 por possuir diagnóstico de DRC, 1 por histórico de IRA e 2 por estarem gestantes. Dos 154 pacientes coletados, 57 não foram incluídos nesse estudo por não terem os resultados de albuminúria.

\section{Grupos do estudo}

Para alocação, os pacientes foram categorizados de acordo com o IMC, subdivididos em dois grupos: obesidade moderada, severa e mórbida (grupo A) e super e super super obesidade (grupo B), com as seguintes faixas de IMC para cada classificação: Grupo A: 30-39,99 kg/m², 40-49 kg/m² e Grupo B: $\geq 50 \mathrm{~kg} / \mathrm{m}^{2}$ e $\geq 60 \mathrm{~kg} / \mathrm{m}^{2}$. Adicionalmente, os indivíduos também foram classificados de acordo com a faixa etária para que fosse relacionado aos fatores de risco para DRC nos pacientes. Foi utilizada a classificação de Ferreira, Szwarcwald \& Damacena (2019) subdivididos em dois grupos: indivíduos de 20 a 39 anos e 40 a 59 anos.

\section{Mensurações}

As coletas de dados ocorreram durante a consulta de Nutrição, por meio de formulário estruturado, com informações: socioeconômicas, clínicas, estilo de vida, antropométricas e laboratoriais. Na oportunidade foram solicitados exames laboratoriais, avaliação nutricional e posteriormente conferido os dados em prontuário.

\section{Escolaridade}

Foi considerada de acordo com o tempo de estudo, de modo que apresentavam baixa escolaridade os indivíduos com tempo de instrução inferior a 4 anos de estudo (Delors et al.1998).

\section{Estilo de Vida}

A partir desses dados foram avaliados os principais fatores de risco para DRC. As informações foram auto referidas sobre: tabagismo e etilismo, que classificou como tabagistas e etilistas aqueles que afirmaram consumir fumo ou bebidas alcoólicas regularmente nos últimos trinta dias, e ex-tabagista e ex-etilista aqueles que já os consumiram alguma vez na vida 
(Kuhneet al. 2009); sedentarismo, sendo aqueles que relataram prática de exercício físico inferior a 150 minutos por semana (Haskell et al. 2007). Foram classificados como portadores de hipertensão (HAS), diabetes mellitus (DM) e doença cardiovascular os pacientes que possuíam diagnóstico médico conforme prontuário. Também foi questionado o uso de medicamentos que contribuem para o tratamento das morbidades envolvidas na obesidade (Márquez, Ruiz-Hurtado, Segura \& Ruilope, 2019) sendo: hipotensores, inibidores da enzima conversora de angiotensina (IECA), betabloqueadores, antagonistas dos receptores da angiotensina, antagonistas do receptor de mineralocorticoide; hipoglicemiantes orais; insulina e estatinas.

\section{Avaliação Antropométrica}

As variáveis peso e altura foram aferidas com auxílio da balança digital Líder® com estadiômetro acoplado, modelo P150C, fabricada no Brasil com capacidade máxima de $300 \mathrm{~kg}$ e precisão de $100 \mathrm{~g}$. Essas medidas foram realizadas segundo técnicas preconizadas por Lohman, Roche \& Martorell (1988). Os valores de peso e altura foram utilizados para o cálculo de IMC, e classificados conforme Requinst (1997).

\section{Mensurações laboratoriais}

Realizados no laboratório de análises clínicas do HUPAA e analisados de acordo com os seguintes métodos: enzimático - glicemia em jejum, glicemia pós-prandial, hemoglobina glicosilada; quimioluminscência - ferritina e cortisol basal coletado entre 07:00 e 09:00; cinético - ureia e creatinina; fórmula de Fredwald - lipidograma completo (colesterol total, lipoproteína de baixa densidade [low-density lipoprotein (LDL)] e lipoproteína de alta densidade [high density lipoprotein $(\mathrm{HDL})]$ ), triglicerídeos; turbidimetria - proteína C reativa (PCR) e albuminuria, sumário de urina; e calculado o clearance de creatinina (Michels et al. 2010). Os pontos de corte e referências dos padrões de normalidade dos referidos exames seguiram as recomendações do laboratório de análise clínica do HUPAA, pela Diretriz Brasileira de Hipertensão (2016), Diretriz Brasileira de Dislipidemias (2017) e Diretriz Brasileira de Diabetes (2019), citados no Quadro 1.

Quadro 1: Valores de referência dos parâmetros laboratoriais utilizados na pesquisa.

\begin{tabular}{|c|c|}
\hline Parâmetros laboratoriais & Valor de referência \\
\hline Glicemia Jejum elevada & $>99 \mathrm{mg} / \mathrm{dl}$ \\
\hline Glicemia Pós-Prandial elevada & $>140 \mathrm{mg} / \mathrm{dl}$ \\
\hline Hemoglobina glicosilada elevada & $>300 \mathrm{ng} / \mathrm{mL} \mathrm{em} \mathrm{homen}$ \\
\hline Ferritina elevada & $>150 \mathrm{a} 200 \mathrm{ng} / \mathrm{mL}$ em mulheres \\
\hline Cortisol Basal elevado & $>19,4 \mathrm{~g} / \mathrm{dL}$ \\
\hline Creatinina séria elevada & $>1,130 \mathrm{mg} / \mathrm{dL}$ em homens \\
\hline Ureia elevada & $>50 \mathrm{mg} / \mathrm{dL}$ \\
\hline Colesterol total elevado & $>190 \mathrm{mg} / \mathrm{dL}$ \\
\hline LDL elevado & $>115 \mathrm{mg} / \mathrm{dL}$ \\
\hline HDL elevado & $<40 \mathrm{mg} / \mathrm{dL}$ \\
\hline Triglicerídeos elevado & $>150 \mathrm{mg} / \mathrm{dL}$ \\
\hline PCR elevado & $>5 \mathrm{mg} / \mathrm{L}$ \\
\hline Albuminúria & $20 \mathrm{e} 300 \mathrm{mg} / \mathrm{g} \mathrm{de} \mathrm{creatinina}$ \\
\hline Clearance de creatinina diminuído & $<60 \mathrm{~mL} / \mathrm{min}$ \\
\hline
\end{tabular}

Fonte: Autores. Os pontos de corte e referências dos padrões de normalidade dos referidos exames seguiram as recomendações da Diretriz Brasileira de Hipertensão (2016), Diretriz Brasileira de Dislipidemias (2017) e Diretriz Brasileira de Diabetes (2019) e de acordo com o laboratório de análise clínica do HUPAA. 


\section{Aspectos éticos}

O presente trabalho foi previamente aprovado pelo Comitê de Ética em Pesquisa da Universidade Federal de Alagoas sob o parecer de número 43975115.9.0000.5013. Todos os participantes assinaram o Termo de Consentimento Livre e Esclarecido.

\section{Análises estatísticas}

Os dados foram processados com o auxílio do software IBM SPSS Statistics for Windows, Version 27.0. Para análise e distribuição dos dados, foi utilizado o teste de Kolmogorov-Sminorv. As variáveis contínuas foram dispostas como médias e desvio padrão quando apresentaram distribuição normal e mediana e intervalo interquartil quando apresentaram distribuição não normal. As variáveis categóricas foram apresentadas através de números absolutos e percentagens. O teste do qui quadrado foi utilizado para: comparação dos parâmetros laboratoriais segundo a presença de albuminúria; comparação da frequência de albuminúria, HAS e DM entre os grupos A e B; comparação dos fatores de risco para DRC com a faixa etária. Para comparação dos exames laboratoriais pelos grupos IMC foi utilizado o teste t e o teste de Mann-Whitney. Também foi realizado a correção de Spearman entre albuminúria versus idade e IMC. Todos os testes foram aplicados com significância estatística em $5 \%(\mathrm{p}<0,05)$ e intervalo de confiança de $95 \%$.

\section{Resultados}

Participaram do estudo 97 indivíduos (Figura 1) na faixa etária de 21 a 59 anos $(43,4 \pm 9,5)$, com IMC de 30,6 a 92,5 $\mathrm{kg} / \mathrm{m}^{2}(48,3 \pm 11,4)$, sendo 84 (86,59\%) do sexo feminino. Destes, 51 (52,5\%) apresentavam união estável e 92 (94,8\%) mais que 4 anos de escolaridade, a média de membros da família por domicilio foi $3 \pm 1,09$ indivíduos, com renda mensal de $\mathrm{R} \$ 1982 \pm 1326$ e renda per capta de $\mathrm{R} \$ 679,3 \pm 512,3$.

Figura 1: Fluxograma dos pacientes inseridos na pesquisa.

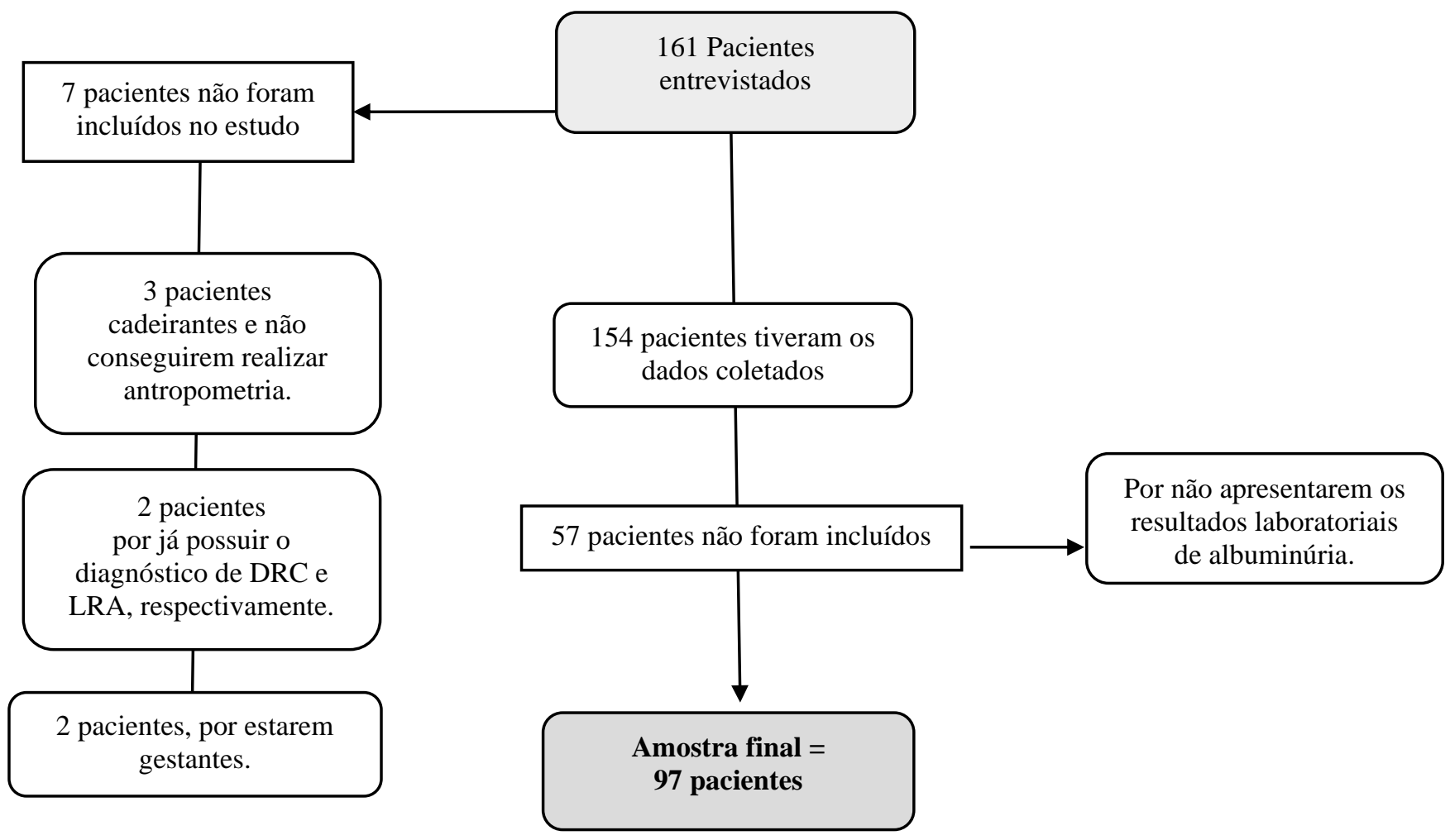

Fonte: Autores. 
Ao analisar o sumário de urina desses obesos, somente $1 \%(\mathrm{n}=1)$ apresentou excreção de proteína na urina. Por outro lado, identificou-se que 21,6\% (n=21) dos indivíduos estavam com albuminúria, com mediana de 36mg/g (média 63,8mg/g/ desvio padrão $55,7 \mathrm{mg} / \mathrm{g}$ )). Não houve associação entre marcadores laboratoriais de rastreio, função e lesão renal, fatores de risco para a DRC e albuminúria levando em consideração a presença e ausência de albuminúria (Tabela 1).

Tabela 1: Associação entre parâmetros laboratoriais e albuminúria em obesos, nos anos de 2018 e 2019, em Maceio-AL.

\begin{tabular}{|c|c|c|c|c|c|}
\hline \multirow{3}{*}{ Parâmetros } & \multicolumn{4}{|c|}{ Albuminúria } & \multirow{3}{*}{ Valor de $p$} \\
\hline & \multicolumn{2}{|c|}{ Sim } & \multicolumn{2}{|c|}{ Não } & \\
\hline & $\mathrm{n}$ & $\%$ & $\mathrm{n}$ & $\%$ & \\
\hline Glicemia de jejum elevada & 10 & 24,4 & 31 & 75,6 & 0,59 \\
\hline HbA1c elevada & 10 & 22,7 & 34 & 77,3 & 0,67 \\
\hline Glicemia pós-prandial elevada & 5 & 20 & 20 & 80 & 0,80 \\
\hline Uremia & 1 & 100 & 0 & 0 & 0,06 \\
\hline Creatinina elevado & 1 & 100 & 0 & 0 & 0,06 \\
\hline Clearance de creatinina diminuído & 1 & 12,5 & 7 & 87,5 & 0,40 \\
\hline Hipertrigliceridemia & 10 & 29,4 & 24 & 70,6 & 0,11 \\
\hline Hipercolesterolemia & 9 & 23,7 & 29 & 76,3 & 0,76 \\
\hline LDL elevado & 10 & 25 & 30 & 75 & 0,60 \\
\hline HDL diminuído & 12 & 31,6 & 26 & 68,4 & 0,09 \\
\hline Ferritina elevada & 11 & 21,2 & 41 & 78,8 & 0,87 \\
\hline PCR elevado & 13 & 27,7 & 34 & 72,3 & 0,41 \\
\hline Cortisol elevado & 8 & 100 & 0 & 0 & 0,13 \\
\hline
\end{tabular}

Teste qui quadrado. Significância estatística $=$ valor de $\mathrm{p}<0,005$. Abreviações: $\mathrm{N}=$ número absoluto; \% percentual; HbA1c=hemoglobina glicada; HDL-c= lipoproteína de alta densidade; LDL-c = lipoproteína de alta densidade; PCR= Proteína C reativa. Referências para avaliação dos parâmetros: Diretriz Brasileira de Hipertensão (2016), Diretriz Brasileira de Dislipidemias (2017), e Diretriz Brasileira de Diabetes (2019).

Fonte: Autores

Sobre o uso medicamentos, foi constatado que 64,4\% $(n=64)$ dos pacientes utilizavam medicamentos hipotensores, $2 \%(n=2)$ IECA e $14,1 \%$ betabloqueadores. 34,3\% $(n=34)$ utilizavam hipoglicemiantes orais; $7,1 \%(n=7)$ faziam uso de insulina; e $16,2 \%(n=16)$ de estatinas.

No que diz respeitos aos grupos A e B, a presença de albuminúria, HAS e DM não diferiu estatisticamente entre os grupos (Tabela 2).

Os parâmetros laboratoriais analisados na Tabela 2, também foram relacionados com o IMC. Dentre os parâmetros laboratoriais analisados, apresentaram significância estatística o HDL $(\mathrm{p}<0,05)$, que apresentou no grupo A média de

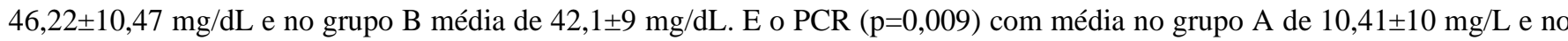

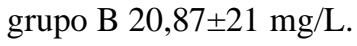


Research, Society and Development, v. 10, n. 12, e316101220490, 2021

(CC BY 4.0) | ISSN 2525-3409 | DOI: http://dx.doi.org/10.33448/rsd-v10i12.20490

Tabela 2: Frequência de albuminúria, hipertensão e diabetes mellitus, de acordo com o IMC em obesos, nos anos de 2018 e 2019, em Maceio-AL.

\begin{tabular}{ccccc}
\hline Parêmetros & Sim & $\begin{array}{c}\text { Grupo A } \\
(\mathrm{n}=60)\end{array}$ & $\begin{array}{c}\text { Grupo B } \\
(\mathrm{n}=38)\end{array}$ & Valor de p \\
\hline Albuminúria & $\mathbf{n}$ & 29 & 42,9 & 0,152 \\
& $\boldsymbol{\%}$ & 54,9 & 9 & \\
HAS & $\mathbf{n}$ & 39 & 45,1 & 0,038 \\
& $\boldsymbol{\%}$ & 74,2 & 32 & 0,073 \\
DM & $\mathbf{n}$ & 23 & 25,8 & \\
& & & & \\
\hline
\end{tabular}

Teste do qui-quadrado. Significância estatística = valor de p<0,005. Abreviações: $\mathrm{N}=$ número absoluto; \% percentual. HAS: Hipertensão arterial sistêmica; DM: Diabetes Mellitus tipo 2.

Fonte: Autores

Foi realizado uma correlação entre albuminúria versus IMC e idade (Tabela 3), porém ambas apresentaram uma fraca correlação.

Tabela 3: Correlação entre albuminúria com IMC e idade.

\begin{tabular}{ccc}
\hline & Albuminúria & \\
& Idade & IMC \\
\hline Coeficiente de correlação & 0,031 & 0,136 \\
Valor de $\mathbf{p}$ & 0,761 & 0,184 \\
\hline
\end{tabular}

Teste não paramétrico. Correlação de Spearman. Significância estatística = valor de $p<0,005$.

Abreviações: IMC $=$ índice de massa corporal.

Fonte: Autores.

Em relação aos demais fatores de risco para desenvolvimento da DRC, foi observado nos obesos uma maior frequência de HAS, sedentarismo e DM2. E ao categorizar de acordo com a faixa etária, apresentaram diferença significativa para HAS no grupo 2 e ex-tabagismo no grupo 1 (Tabela 4). 
Research, Society and Development, v. 10, n. 12, e316101220490, 2021

(CC BY 4.0) | ISSN 2525-3409 | DOI: http://dx.doi.org/10.33448/rsd-v10i12.20490

Tabela 4: Fatores de risco para o desenvolvimento da DRC e a associação com faixa etária em obesos, nos anos de 2018 e 2019, em Maceio-AL.

\begin{tabular}{ccccc}
\hline Fatores & $\mathbf{2 0}$ a 39 anos & \multicolumn{4}{c}{$\mathbf{4 0}$ a 59 anos } & Valor p \\
$\mathrm{n}=34$ & $\%$ & $\mathrm{n}=63$ & $\%$
\end{tabular}

\begin{tabular}{|c|c|c|c|c|c|}
\hline \multicolumn{6}{|c|}{ HAS } \\
\hline Sim & 20 & 58,8 & 51 & 81,0 & $0,01 *$ \\
\hline Não & 14 & 41,2 & 12 & 19,0 & \\
\hline \multicolumn{6}{|l|}{ DM } \\
\hline Sim & 8 & 23,5 & 23 & 36,5 & 0,33 \\
\hline Não & 26 & 76,5 & 40 & 63,5 & \\
\hline \multicolumn{6}{|c|}{ HAS + DM } \\
\hline Sim & 5 & 14,7 & 19 & 30,2 & 0,20 \\
\hline Não & 29 & 85,3 & 44 & 69,8 & \\
\hline \multicolumn{6}{|c|}{ DCV } \\
\hline Sim & 1 & 2,9 & 1 & 1,6 & 0,89 \\
\hline Não & 33 & 97,1 & 62 & 98,4 & \\
\hline
\end{tabular}

Sedentarismo

$\begin{array}{llllll}\text { Sim } & 18 & 52,9 & 43 & 68,3 & 0,26 \\ \text { Não } & 16 & 47,1 & 20 & 31,7\end{array}$

Tabagismo

$\begin{array}{lccccc}\text { Sim } & 3 & 8,8 & 3 & 4,8 & 0,72 \\ \text { Não } & 31 & 91,2 & 58 & 92,1\end{array}$

\section{Ex-tabagismo}

$\operatorname{Sim}$

$1,8 \quad 7$

Não

$30 \quad 88,2$

55

87,3

\section{Etilismo}

Sim

$9 \quad 26,5$

12

19,0

0,64

Não

$25 \quad 73,5$

50

79,4

\section{Ex-etilismo}

$\begin{array}{lccccc}\text { Sim } & 1 & 2,9 & 3 & 4,8 & 0,87 \\ \text { Não } & 33 & 97,1 & 58 & 92,1\end{array}$

Teste qui quadrado. *Significância estatística $=$ valor de $\mathrm{p}<0,005$. Abreviações: $\mathrm{n}=$ número absoluto; \% percentual; HAS = hipertensão arterial sistêmica; $\mathrm{DM}=$ diabetes mellitus tipo $2 ; \mathrm{DCV}=$ doença cardiovascular.

Fonte: Autores. 


\section{Discussão}

Nessa pesquisa, destacou-se a elevada frequência de albuminúria em obesos, independentemente do grau da obesidade. Além disso, indivíduos de maior faixa etária, mas não idosos, destacaram-se pela presença de HAS e baixos níveis de HDL, destacando como um fator de risco cardiovascular, e subsequente para DRC.

A obesidade grave é fator de risco para várias doenças crônicas, incluindo a DRC (Unamuno et al. 2018; Kovesdy, Furth \& Zoccali, 2017). Além disso, o excesso de TA se relaciona independentemente com modificações no organismo, atribuídas a produção de adipocinas, e quando secretadas demasiadamente levam a desarmonia da homeostase e inflamação crônica de baixo grau (Pellegrinelli, Carobbio \& Vidal-Puig, 2016).

Em obesos, a identificação da albuminúria pode ser um útil aliado na avaliação do risco de desenvolvimento de DRC, e a observação nesse estudo de sua frequência elevada (21,6\%) foi considerado um fator de extrema relevância. Este achado foi superior ao evidenciado por Rosenstock et al. 2018 (13,3\%), Minoo et al. 2015 (11,8\%) e Bhatt et al. 2019 (11,66\%), em pesquisas semelhantes com obesos realizadas em Nova York, Teerã e na Índia.

Alguns fatores podem contribuir para a menor ocorrência de albuminúria, como descrito por esses autores citados acima. Rosenstock et al. 2018, por exemplo, realizou a pesquisa com obesos no momento da internação para realização da cirurgia bariátrica, de modo que, é possível que a perda de peso necessária para realização da cirurgia, juntamente com a utilização de IECA e bloqueadores do receptor da angiotensina (Márquez et al. 2019) tenham impactado na redução dos níveis de albuminúria. Minoo et al. (2015), por sua vez, não incluiu portadores de HAS e DM, fatores que deterioram a função renal (Bastos, Bregman \& Kirsztajn, 2010). E Bhatt et al. 2019, além de não incluir pacientes com HAS e DM, incluiu indivíduos com IMC na faixa de 25,0-29,9 kg/m², os quais não foram incluídos no presente estudo.

Alterações glicêmicas e no perfil lipídico são comumente observadas em obesos, devido ao estado inflamatório, resistência à insulina e hábitos alimentares inadequados (Engin \& Engin, 2017; Pellegrinelli, Carobbio \& Vidal-Puig, 2016; Barroso et al. 2017) e também se relacionam com a albuminúria (Bastos, Bregman \& Kirsztajn, 2010; Minoo et al. 2015). Os achados desse estudo além de não terem mostrado diferença significativa entre essas variáveis, destacou que metade dos indivíduos estudados apresentava normalidade nos níveis glicêmicos e no perfil lipídico, apesar da obesidade. É provável que o acompanhamento medicamentoso e dietético (Márquez et al. 2019), tenha refletido em tais achados.

A obesidade por si só é capaz de sobrecarregar a função renal e ocasionar albuminúria. O aumento de massa corporal, sem o aumento correspondente no número de néfrons, leva a um aumento no fluxo plasmático renal e do volume de filtração glomerular, ocasionando uma síndrome de hiperfiltração glomerular (Futrakul, Sridama \& Futrakul, 2009; Navarro \& Ardiles, 2015), além dos mecanismos envolvidos com a adiposidade, levam a lesão endotelial, hipertensão glomerular e/ou lesão de podócitos, induzindo a uma lesão endotelial glomerular. Ambas as formas podem levar ao vazamento de albumina na urina (Futrakul, Sridama \& Futrakul, 2009).

Nesse estudo, as médias de idade e IMC entre os grupos avaliados foram semelhantes as obtidas em outros estudos com obesos, como Rosenstock et al. 2018, que obteve respectivamente 42,1 $\pm 11,3$ anos e 43,9 $\pm 8,1 \mathrm{~kg} / \mathrm{m}^{2}$ e também ao de Brix et al. 2019 com $40 \pm 12$ anos e 45,6 $\pm 6,6 \mathrm{~kg} / \mathrm{m}^{2}$, predominando os obesos graves.

O IMC elevado ocasiona uma cascata de reações no organismo, derivados do TA e da lipotoxicidade, de modo que o maior peso corporal está associado, por exemplo, a menores níveis de HDL (Rosenstock et al. 2018), uma lipoproteína relacionada a funções antiaterogênicas, que exerce atividades antioxidantes e anti-inflamatórias, devido ao excesso de gordura visceral prejudicar os níveis de lipoproteínas, atividade antioxidante e funcionalidade das lipoproteínas (Park et al. 2018). E, isto foi observado no presente estudo entre os indivíduos de ambos os grupos estudados. Além disso, os níveis séricos de PCR desses indivíduos também se destacaram, provavelmente pela maior quantidade de TA (Engin \& Engin, 2017) exacerbar a produção de citocinas pró-inflamatórias. 
A albuminúria também tem se associado com o avançar da idade (Teliewubai et al. 2020), além de sua associação com a presença de doenças crônicas, fatores de risco já reconhecidos para DRC. No entanto, os resultados observados nesse estudo não foram de encontro aos presentes na literatura, possivelmente pelo grupo avaliado ser basicamente composto por adultos jovens.

A obesidade também apresenta complexa relação com a fisiopatologia da HAS e DM. O TA participa de forma ativa do metabolismo endócrino, além de secretar adipocinas inflamatórias que tem papel fundamental na gênese da resistência periférica a insulina (Barroso et al. 2017; Fantin et al. 2019). Somado a isso, o avançar da idade intensifica esses processos, além de redistribuir a composição adiposa para locais ectópicos (Jura \& Kozak, 2016). Nas características de base do nosso estudo evidenciamos prevalência importante de HAS e DM nos pacientes obesos graves, com destaque para os adultos de meia idade.

No quesito socioeconômico, esses participantes possuem uma renda média superior aos usuários do SUS da região (Silva, Cabral Junior \& Vasconcelos, 2010). Esta diferenciação social pode ter sido observada, provavelmente, pela maior parte dos indivíduos, deste estudo, pertencerem a um programa de cirurgia bariátrica, que gera custos financeiros para os usuários durante o tratamento, pois apenas o procedimento cirúrgico é custeado pelo governo, fazendo com que os usuários mais carentes tenham dificuldade para adesão.

\section{Conclusão}

Obesos apresentaram elevada frequência de albuminúria, sugerindo que somente a obesidade, independentemente de sua gravidade, é capaz de aumentar a morbimortalidade nesses pacientes. Adicionalmente, indivíduos de maior faixa etária possuíam maior prevalência de HAS e menor nível sérico de HDL, consequentemente maior risco de desenvolvimento de DRC. É importante a realização de mais estudos para identificar os fatores que foram preponderantes para o maior risco de desenvolvimento de microalbuminúria em obesos, e tentar demonstrar por que os indivíduos com IMC grau I e II apresentaram maior prevalência do que quando comparados com obesos graves.

\section{Agradecimentos}

Ao apoio da Coordenação de Aperfeiçoamento de Pessoal de Nível Superior - Brasil (CAPES).

\section{Referências}

Barroso, T. A., Marins, L. B., Alves, R., Gonçalves, A. C. S., Barroso, S. G., \& Rocha, G. S. (2017). Associação entre a obesidade central e a incidência de doenças e fatores de risco cardiovascular. Int J Cardiovasc Sci. 30 (5):416-24.

Bastos, M. G., Bregman, R., \& Kirsztajn, G. M. (2010). Doença renal crônica: frequente e grave, mas também prevenível e tratável. Rev Assoc Med Bras. 56 (2):248-53.

Bhatt, V. R., Khese, V. B., Jadhav, S. L., \& Kakrani, A. L. (2019). Urinary albumin excretion, estimated glomerular filtration rate, and prevalence of microalbuminuria in obese nondiabetic and nonhypertensive adults: A cross-sectional study. Indian J Nephrol. 29(3):166-71.

Brix, J. M., Herz, C. T., Kopp, H. P., Feder, A., Krzizek, E. C., Sperker, C., et al. (2019). Albuminuria in Patients with Morbid Obesity and the Effect of Weight Loss Following Bariatric Surgery. Obes Surg. 29(11):3581-8.

Chagnac, A., Weinstein, T., Korzets, A., Ramadan, E., Hirsch, J., \& Gafter, U. (2000). Glomerular hemodynamics in severe obesity. Am J Physiol Renal Physiol. 278(5):817-22.

Delors, J., Al-Mufti, I., Amagi, I., Carneiro, R., Chung, F., Geremek, B. et al. Educação: um tesouro a descobrir: relatório para a UNESCO da Comissão Internacional sobre Educação para o Século XXI. Brasília: UNESCO, 1998.

Ejerblad, E., Fored, C. M., Lindblad, P., Fryzek, J., McLaughlin, J. K., \& Nyrén, O. (2006) Obesity and risk for chronic renal failure. J Am Soc Nephrology, 17(6):1695-1702.

Engin, A. B. \& Engin, A. (2017) The pathogenesis of obesity-associated adipose tissue inflammation. Obesity and Lipotoxicity. Adv Exp Med Biol. 960:22145 . 
Faludi, A. A., Izar, M. C. O., Saraiva, J. F. K., Chacra, A. P. M., Bianco, H. T., Afiune, Neto, A. et al. (2017). Atualização da Diretriz Brasileira de Dislipidemias e Prevenção da Aterosclerose - 2017. Arq Bras Cardiol. 9(2) 1:1-76.

Fantin, F., Giani, A., Zoico, E., Rossi, A. P., Mazzali, G., \& Zamboni, M. (2019). Weight Loss and Hypertension in Obese Subjects. Nutrients. 11(7):1667.

Ferreira, A. P. S., Szwarcwald, C. L., \& Damacena, G. N. (2019). Prevalência e fatores associados da obesidade na população brasileira: estudo com dados aferidos da Pesquisa Nacional de Saúde, 2013. Rev Bras Epidemiol. (22):e190024.

Futrakul, N., Sridama, V., \& Futrakul, P. (2009). Microalbuminuria - a biomarker of renal microvascular disease. Ren Fail. 31(2):140-3.

Haskell, W. L., Lee, I. M., Pate, R. R., Powell, K. E., Blair, S. N., Franklin, B. A., et al. (2007). Physical activity and public health: updated recommendation for adults from the American College of Sports Medicine and the American Heart Association. Med Sci Sports Exerc. 39(8):1423-34.

Herrington, W. G., Smith, M., Bankhead, C., Matsushita, K., Stevens, S., Holt, T., et al. (2017). Body-mass index and risk of advanced chronic kidney disease: prospective analyses from a primary care cohort of 1.4 million adults in England. PloS One, 12(3): e0173515.

Jura, M., Kozak, L. P. (2016). Obesity and related consequences to ageing. Age (Dordr), 38(1):23.

Kovesdy, C., Furth, S., Zoccali, C. (2017). World Kidney Day Steering Committee. Obesity and kidney disease: hidden consequences of the epidemic. J Bras Nefrol. 39(1):260-2.

Kuhnen, M., Boing, A. F., Oliveira, M. C. D., Longo, G. Z., Njaine, K. (2009). Tabagismo e fatores associados em adultos: um estudo de base populacional. Rev Bras Epidemiol. 12(4):615-26.

Lohman, T. G., Roche, A. F, Martorell, R. (1988). Anthropometric standardization reference manual. Champaign: Human Kinetics Books.

Malachias, M. V. B., Souza, W. K. S. B., Plavnik, F. L., Rodrigues, C. I. S., Brandão, A. A., Neves, M. F. T. (2016). $7^{\circ}$ Diretriz Brasileira e Hipertensão Arterial. Arq Bras Cardiol. 107(3):1-103.

Márquez, D.F., Ruiz-Hurtado, G. Segura, J. Ruilope, L. (2019). Microalbuminuria and cardiorenal risk: old and new evidence in different populations. F1000Research, 8, F1000 Faculty Rev-1659.

Michels, W. M., Grootendorst, D. C., Verduijn, M., Elliott, E. G., Dekker, F. W., Krediet, R. T. (2010). Performance of Cockcroft-Gault, MDRD and CKDEPI formulas in relation to GFR, age and body size. Clin J Am Soc Nephrol. 5(6):1003-9.

Minoo, F., Mahdavi-Mazdeh, M., Abbasi, M. R., Sohrabi, S. (2015). Impact of the severity of obesity on microalbuminuria in obese normotensive nondiabetic individuals. J Renal Inj Prev. 4(2):34-8.

Navarro, G., Ardiles, L. (2015). Association between obesity and chronic renal disease. Rev Med Chile, 143(1):77-84.

Park, K. H., Yadav, D., Kim, S. J., Kim, J. R., Cho, K. H. (2018). Slim body weight is highly associated with enhanced lipoprotein functionality, higher HDLC, and large HDL particle size in young women. Front Endocrinol. 9:406.

Pellegrinelli, V., Carobbio, S., Vidal-Puig, A. (2016) Adipose tissue plasticity: how fat depots respond differently to pathophysiological cues. Diabetologia, 59 (6): $1075-88$.

Pinto-Sietsma, S. J., Navis, G., Janssen, W. M., Zeeuw, D., Gans, R. O, Jong, P. E. (2003). PREVEND Study Group. A central body fat distribution is related to renal function impairment, even in lean subjects. Am J Kidney Diseases,41(4):733-41.

Renquist, K. (1997). Obesity classification. Obes Surgery, 7(6):523.

Rosenstock, J. L., Pommier, M., Stoffels, G., Patel, S., Michelis, M. F. (2018). Prevalence of proteinuria and albuminuria in an obese population and associated risk factors. Front Med. 5:122.

Serra, A., Romero, R., Lopez, D., Navarro, M., Esteve, A., Perez, N., et al. (2008) Renal injury in the extremely obese patients with normal renal function. Kidney Int. 2008;73(8):947-55.

Silva, P. M. C., Cabral Junior, C. R., \& Vasconcelos, S. M. L. (2010). Ingestão do cálcio na obesidade de mulheres atendidas pelo Sistema Único de Saúde. Rev Nutr. 23(3):357-67.

Sociedade Brasileira de Diabetes (SBD). (2019). Diretrizes da Sociedade Brasileira de Diabetes 2019-2020. Sociedade Brasileira de Diabetes, p.1-491.

Stenvinkel, P., Zoccali, C., \& Ikizler, T. A. (2013). Obesity in CKD - what should nephrologists know? J Am Soc Nephrology, 24 (11):1727-36.

Teliewubai, J., Fan, X., Ji, H., Maimaitiaili, R., Tang, J., Zhao, S., et al. (2020). Does healthy obesity exist in the elderly? Findings from the Northern Shanghai Study. Nutr Metab Cardiovasc Dis. 30(5):749-57.

Unamuno, X., Gómez-Ambrosi, J., Rodríguez, A., Becerril, S., Frühbeck, G., \& Catalán, V. (2018) Adipokine dysregulation and adipose tissue inflammation in human obesity. Eur J Clin Invest. 48(9): e12997.

Valensi, P., Assayag, M., Busby, M., Paries, J., Lormeau, B., \& Attali, J.R. Microalbuminuria in obese patients with or without hypertension. Int J Obes Relat Metab Disord. 1996;20(6):574-9.

WHO Expert Consultation. (2004). Appropriate body-mass index for Asian populations and its implications for policy and intervention strategies. Lancet, 363 (9403):157-63. 
Research, Society and Development, v. 10, n. 12, e316101220490, 2021

(CC BY 4.0) | ISSN 2525-3409 | DOI: http://dx.doi.org/10.33448/rsd-v10i12.20490

Yazdani, N., Sharif, F., Elahi, N., Hosseini, S.V., \& Ebadi, A. (2019). Exploration of the Quality of Life in Iranian Morbid Obese People: A Qualitative study. Int J Community Based Nurs Midwifery, 7(2):138-49.

Zanella, M. T. Microalbuminúria: fator de risco cardiovascular e subestimado na prática clínica. (2006). Arq Bras Endocrinol Metab. 50(2):313-21. 